\section{Goethe oder Newton oder Beide?}

Von L. HofrmanN.

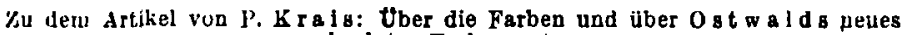
absolutes Farbensystem.

(Eingeg. 25./4. 1918.)

$P$ a a l $\mathrm{Kra}$ is hat in dieser Zeitschrift ${ }^{1}$ ) einen Artikel geschrieben, der für mich Anla B ist, dasselbe Thema hier anzuschneiden. Ich sende voraus, daß ich für Goe thes Farbenlehre bin und ein "Lexikon für seine Richtigkeit" geschrieben habe. $\mathrm{K} r$ a is hat das absolute Farbensystem von $\mathrm{Ostwald}$ in sehr rühmender und anerkennenswerter Weise besprochen, und ich schließe mich ihm vollkommen an, was Einteilung der Farbencharaktere, nach a) Helligkeit, b) Reinheit und c) Farbton betrifft. Mit dem Instrument "Pomi" und der "Grauskala" hatte ich bis jetzt noch nicht Gelegenheit, Prohen anzustellen, schließe mich aber gern der Autorität von $\mathrm{K} \mathbf{r}$ a is an.

Nun zu der Streitfrage: Dèn Anerkennungsbestrebungen für Goethe, die in München zutage traten, stehe ich ganz fern. Ich kenne sie auch jetzt noch nur teilweise und halte mich darüber hier gar nicht auf.

$\mathrm{K} r \mathrm{r}$ is nimmt den vermittelnden Standpunkt ein, den Prof. Ra ehl mann im Jahrb. d. Goethegesellschaft Bd. 3, 1916 eingenommen, und den $\mathrm{Ostwald}$, namentlich in seinem schönen, sonst von mir sehr geschätzten Buche „Malerbriefe" und auch Prof. B e r g e $r$ in den Münchener kunsttechnischen Blättern einnehmen. - Os tw a ld gibt im fünften Briefe seines Buches eine Erklärung nach $\mathrm{New}$ t on $\mathrm{s}$ Theorie und im achten eine solche mit „durchscheinenden, weißen oder trüben Mitteln", nach G o e th e. - Das halte ich nicht nur für unstatthaft, sondern für eine wissenschaftliche Unmöglichkeit.

Es kann nicht darauf ankommen, hier sofort damit den Beweis antreten zu wollen, daß alle Meinungsverschiedenheiten etwa in der Frage des Ionenspektrums - in der $\mathrm{K} \mathrm{r}$ a is ein so heiteres, treffendes Bild mit dem Klaviermonstrum geschaffen hat - zusammengefaßt werden. Der Kampfplatz, auf den Go e t h e herausgefordert hat, liegt auch nicht hier, und ich denke mir eine Lösung der Spektralfrage in G o e $t \mathrm{~h}$ e s Sinn nicht in hoffnungsloser Ferne.

Wichtig ist hier, was $\mathrm{Kr}$ a is über Licht und Farbe mitteilt, und ich führe einige Sätze an, ohne dann weiter darauf einzugehen. „Alles Licht, das nicht rein weiß oder rein (neutral) grau ist, erscheint gefärbt.... Es gibt keine natürliche Lichtquelle, die uns in diesem Sinne rein weißes Licht spendet.... Eine Annäherung wäre dadurch möglich, daß man komplementäre Paare oder Trios, Quartette usw. gleich intensiver Spektralfarbenbündel mischt.... Fine Hauptfarbe müssen wir uns spektralanalytisch nicht etwa als cine Linie, sondern als ein mehr oder weniger breites Band vorstellen."

Nun zur Beantwortung der in der Überschrift genannten Streitfrage: Obschon sonst kein Freund von $S \mathrm{ch}$ ope $\mathrm{n}$ h a u ers Theorie, stehe ich doch hier ganz auf seinem Boden, daß es in. einer abgerundeten Sache (wie es die Farbenlehre ist) nur e in e theoretische Wahrheit geben kann. Tritt noch eine zweite Theorie auf, so kann sie nur feindlich sein, und es muß so lange ein Ringen erfolgen, bis eine unterliegt. Eine Aussöhnung ist ausgeschlossen.

1. N e w t o n sagt: Die Sonne bringt weißes Licht, dus die Farben in sich einschließt, fertig herab zu der Erde. Durch ein Prisma wird der weiße Strahl derart gespalten, daß jeder Farbenstrahl in seiner eigenen Schwingungsgröße heraustritt. Durch die Linse können aber' liese Strahlen wieder gesammelt und zu einem einzigen, weißen, vereinigt werden. - Finzige Ursache ist bei $\mathrm{New}$ to n die Lichtbrechung. (Kniclsung und Biegung kommt hier nicht in Frage.)

2. Go e the sagt: Farbiges licht kommt übcrhaupt nicht in einen weißen Strahl, sondern Farbe entstebt, bildet sich erst auf der Erde, durch den Gegensatz von Licht und Finsternis. Fs ist ein Kampf, ein Ringen von zwei Kräften, und dringt die Finsternis vor gegen das Licht, am Prismarand oder sonstwo, so entsteht Blau, im entgegengesetzten Falle Gelb. Diese beiden sind die einzigen Urfarben. Ihre Steigerung gibt Rot, und ihre Mischung Grün, den Elcmentarfarbenring.

Zur Präzisierung meines Standpunktes möge das unter 1. und 2. Genannte als genügend angenommen werden.

Es ist gewiB sehr löblich, G o e th es Farbenlehre, die man nicht absolut verwerfen kann, für bequem liegende Fälle aus Utilitätsrücksichten, etwa nebenbei heranzuziehen und von Ausgleich zu reden. Aber das ist ganz zu verwerfen. Das Beispiel

1) Angew. Chem. 29, I, 129-131 [1916].
Goethes zeigt dus am besten. Goothe wollte, ohne $\mathrm{New}$ t on direkt anzugreifen, eine andere Farbentheorie einführen, und er hätte die abstrakte $\mathrm{Ne}$ w t o $\mathrm{n}$ sche unberührt in der Dunkelkammer gelassen. Das ganze erste Buch von den fünf Bänden seiner Farbenlehre beweist dies. Aber der Gegensatz machte sich derart geltend, daß G o e th e auf den Kampfplatz mußte, und hier galt es ein Ringen um Sein oder Nichtsein. Wenn man G o e t h e s Farbenlehre studiert, ohne diesen Gesichtspunkt zu beräcksichtigen, so geht es einem leicht, wie es dem großen Physiologen und Physiker Helmholtz dabei ergangen ist: Er fand zwar Goethes Experimente vollkommen richtig, hielt aber die scharfen Angriffe auf $\mathrm{N}$ e w t on für unverständlich. Allein, $G$ o e $t \mathrm{~b}$ e wußte genau, was in der Sache nötig war. G o e th e ist nie mit der Streitlust aufgetreten wie etwa Lessing. Aber hier mußte "gerauft" werden, von ihm, und er hat das, in seiner Weise, auch reichlich begründet. Goethes Farbenlehre ist aber auch als Theorie so begründet und ausgebaut, daß sich die neuesten elektromagnetischen Lichttheorien mit ihr vereinigen lassen, während sie der $\mathrm{New}$ t o n schen "S trah len le hr e", die schon G o e th e bekämpft hat, Berechtigung absprechen.

[A. 54.]

\section{Zur Kenntnis der technisch - wirtschaftlichen chemischen Literatur in England ${ }^{1}$ ).}

Von Prof. Dr. H. Grossmann, Berlin. (Eingeg. 19./4. 1918.)

Nachdem in dieser Zeitschrif $\mathrm{t}^{2}$ ) und in den Berichten der Deutschen Chemischen Gesellschaft ${ }^{3}$ ) unlängst ein neuer Entwurf ausgearbeitet worden ist, der eine Vereinheitlichung der chemischen Literatur und ihren Ausbau besonders in technischer Richtung herbeiführen soll, erscheint es vielleicht nicht ohne Interesse, auf die beachtenswerten Bestrebungen aufmerksam zu machen, die in England, zweifellos zum Teil beeinflußt durch die Zeitschrift für angewandte Chemie, neuerdings zu praktischen Ergebnissen geführt haben. Bei derartigen Bestrebungen wird man sich sagen müssen, daB es sich allgemein empfehlen dürtte, auch vom Feinde zu lernen, wobei es selbstverständlich auch durchaus zweckmäßig erscheint, unumwunden anzuerkenuen, was im feindlichen Auslande geleistet worden ist. Fritz Mauthner hat kürzlich in einem interessanten Aufsatz üher den großen englischen Schriftsteller Jon a th a n $S$ w if $t$, übrigens einer der schärfsten Kritiker Englands, der wohl jemals gelebt hat, im Berliner Tageblatt vom 14./4. darauf hingewiesen, ,daß der Haß gegen England zu einer Gefahr für den deutschen Geist worden wïrde, wenu or den Wissenden zu einer Lügo verführte. "Wir wollen deshalb jedenfalls in der chemischen Literatur diesen Fehler tunlichst vermeiden, wie wir das ja bisher stets getan haben, und bei aller Schärfe der politischen und wirtschaftlichen Gegensätze ruhig anerkennen, was Anerkennung verdient. Selbstverständlich wollen wir aber auch für unsere eigenen Zwecke und Interessen aus den Austrengungen unserer Gegner zu lernen suchen, um sie nach Möglichkcit auf diesem wic auf anderen Gebieten zu übertreffen. $\left.{ }^{4}\right)$

Die neue Organisation, welche die Society of Chemical Industry seit dem Beginn dieses Jahres ihrer Zeitschrift gegeben hat, crscheint mir jedenfalls einer derartig unumechränkten Anerkennung durchuus würdig zu sein. Man hat hier ähnlich wie in der Zeitschrift für angewandte Chemie eine Dreiteilung des Inhalts durchgeführt. So bringt das Journal of Society of Chemical Industry erstmalig in seinem 37. Jahrgang 1918 drei besonders paginierte Teile unter dem Titel: „Review“ (R), „Transactions" (T) und „Abstracts and Patent Literature" (A). Für unsere Interessen erscheint nun von besonderer Bedeutung die Abteilung „R e vi e w", die nach den drei zurzeit vorliegenden Heften des derzeitigen Jahrgangs vom 15. und 31. Ja. nuar sowie vom 15. Februar 1918 nach jeder Richtung hin besonders ausgestaltet worden ist. Die beiden folgenden Teile "Abhandlungen" und „Referate" weisen dagegen gegen die früheren Bände keine we. sentlichen Unterschiede auf. Es erscheint aber vielleicht nicht ohne Interesse, einige Bemerkungen über die Einführung jener neuen Rubrik in das Journal of the Society of Chemical Industry an der Hand der in der Nr. 1 vom 15. Januar gemachten Ausführungen der Redaktion wiederzugeben. Es heißt dort u. a., daß der Vorstand der Gesell-

1) Vorgetragen im Märkischen Bezirksverein am 15./4. 1918.

2) Angew. Chem. 31, III, 175 [1918].

3) Ber. 51, 497 ff. [1918].

-) Vergleiche auch die Einleitung zu der Schrift Th. Schu c h a rd t, ,die Außenhandelsförderung Englands in ihrer neuesten Entwicklung“. Berlin 1918. Deutscher Uberseedienst. 